\title{
NASA's launch communications ground segment for the 21st century Florida spaceport
}

\author{
Christopher J. Roberts, ${ }^{1}$ David R. McCormick, ${ }^{2}$ Robert N. Tye, ${ }^{3}$ Eric J. Harris, ${ }^{4}$ David L. Carter, ${ }^{5}$ and John J. \\ Hudiburg ${ }^{6}$ \\ NASA Goddard Space Flight Center, Greenbelt, MD 20771, USA \\ Patricia H. Peskett, ${ }^{7}$ \\ Peraton Corporation, Greenbelt, Maryland, 20771, USA \\ and \\ Peter B. Celeste, ${ }^{8}$ and Patricia R. Perrotto ${ }^{9}$ \\ Booz Allen Hamilton, Annapolis Junction, MD 20701, USA
}

\begin{abstract}
The National Aeronautics and Space Administration (NASA) Near Earth Network (NEN) Project is implementing a new launch communications ground segment to provide services for the next generation of human and robotic space exploration systems. It will deliver unique and advanced capabilities to accelerate the transformation of Kennedy Space Center into a multiuser spaceport in cooperation with the United States Air Force. The project has leveraged commercial technologies and remote operations concepts matured in NASA's orbiting satellite ground systems to achieve dramatic lifecycle cost efficiencies as compared to the space shuttleera ground segment. The purpose of this paper is to discuss the development history, capabilities, and anticipated use cases of the NEN Launch Communications Segment (LCS).
\end{abstract}

\section{I. Introduction}

$\mathrm{O}$ N July 8, 2011 the National Aeronautics and Space Administration (NASA) Near Earth Network (NEN) S-band antennas at Merritt Island Launch Annex (MILA) and Ponce de Leon (PDL) data and tracking stations provided communications support to the launch and ascent of the final Space Shuttle mission, Space Transportation System135 (STS-135), as it lifted off from the Kennedy Space Center (KSC) Launch Complex 39. Just over twelve days later MILA supported the orbiter Atlantis as it made its final landing on Runway 15 at KSC. Established in 1966, MILA's first mission was the Apollo/Saturn-203 in July of that year. Following the Apollo Program, the PDL station, located 64 kilometers $(\mathrm{km})$ north of MILA, was established in 1979 to ensure continuous communications coverage to the Shuttle during a brief period on ascent when MILA would be unable to close the communications link due to plume attenuation caused by the Shuttle's Solid Rocket Booster (SRB) exhaust. The end of the Shuttle Program brought with it the end of MILA as an operational ground station for NASA's human spaceflight program. MILA was decommissioned in 2011, and PDL was placed into caretaker status. Today, the NEN is completing development of two new modernized ground stations, one located at a new site on KSC property and another at the PDL site [1]. Together they will provide communications support to the next generation of human and robotic space exploration systems, provide additional assets for on-orbit support to the NEN, and serve as a proving ground for future NEN concepts and technology development.

${ }^{1}$ LCS Development Manager, Near Earth Network Project, AIAA Member.

${ }^{2}$ LCS Mission Integration \& Operations Manager, Near Earth Network Project.

${ }^{3}$ LCS Chief Engineer, Near Earth Network Project.

${ }^{4}$ LCS Test Director, Near Earth Network Project.

${ }_{6}^{5}$ Project Manager, Near Earth Network Project.

${ }^{6}$ Mission Commitment \& Integration Manager, Space Communications and Navigation Program, AIAA Member.

${ }^{7}$ Space Communications and Network Services Contract Engineering Manager, Peraton Corporation.

${ }^{8}$ Senior Lead Engineer, Booz Allen Hamilton Aerospace Market.

${ }^{9}$ Lead Engineer, Booz Allen Hamilton Aerospace Market. 

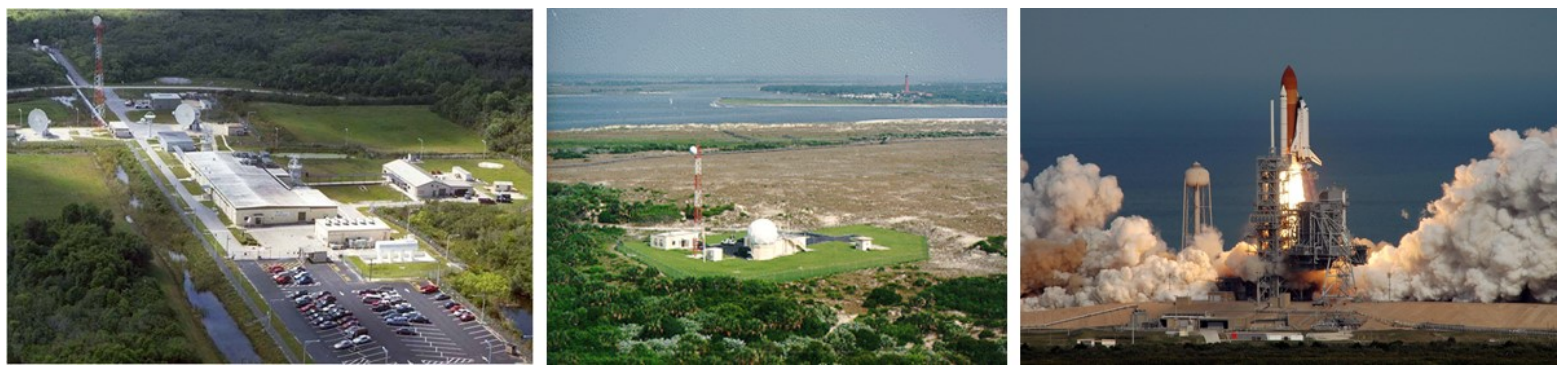

Fig. 1 Left to right: (a) MILA, (b) PDL, and (c) Shuttle launch

The NEN is composed of a set of worldwide ground stations, as shown in Fig. 2, and provides tracking, command, and telemetry space communications services to customer missions operating at various orbits, including low-earth, highly elliptical, geosynchronous, and lunar orbits. The NEN is managed by the Goddard Space Flight Center (GSFC) in Greenbelt, Maryland. It serves as one of NASA's three space communications networks, along with the Space Network (SN) and the Deep Space Network (DSN), under the Space Communications and Navigation (SCaN) Program. The NEN traces its organizational roots to the origin of human space flight for the United States [2]. The Manned Space Flight Network (MSFN) was a worldwide network of equatorial and mid-latitude ground stations that provided tracking, telemetry, commanding and voice communications to the Mercury, Gemini, and Apollo human space flight programs. In the 1970s, NASA merged the MSFN with its robotic satellite communications network, the Satellite Tracking and Data Acquisition Network (STADAN), forming the Spaceflight Tracking and Data Network (STDN). The STDN supported the communications needs of both human and robotic spaceflight missions, including the early space shuttle flights. In the 1980s, NASA began deploying geosynchronous communications satellites and associated ground systems known today as the SN. This led to a reduction in the required number of equatorial and mid-latitude ground station nodes within the STDN. Additionally, the 1990s saw a large increase in science data requirements to support polar-orbiting earth observation satellites, resulting in the establishment of several highlatitude stations. The STDN became the Ground Network, which included NASA owned and operated stations as well as communications service agreements with international partners and emerging commercial ground station providers. The Ground Network became the Near Earth Network in 2008 to better reflect its evolving capabilities to support missions within 2 million $\mathrm{km}$ of Earth, which is where the Federal Communications Commission (FCC) and International Telecommunication Union (ITU) define the end of near-Earth Space. These evolving capabilities included the addition of 18-meter Ka-band antennae at White Sands, New Mexico in support of the Solar Dynamics Observatory (SDO) in geosynchronous orbit and the Lunar Reconnaissance Orbiter (LRO) in lunar orbit. 


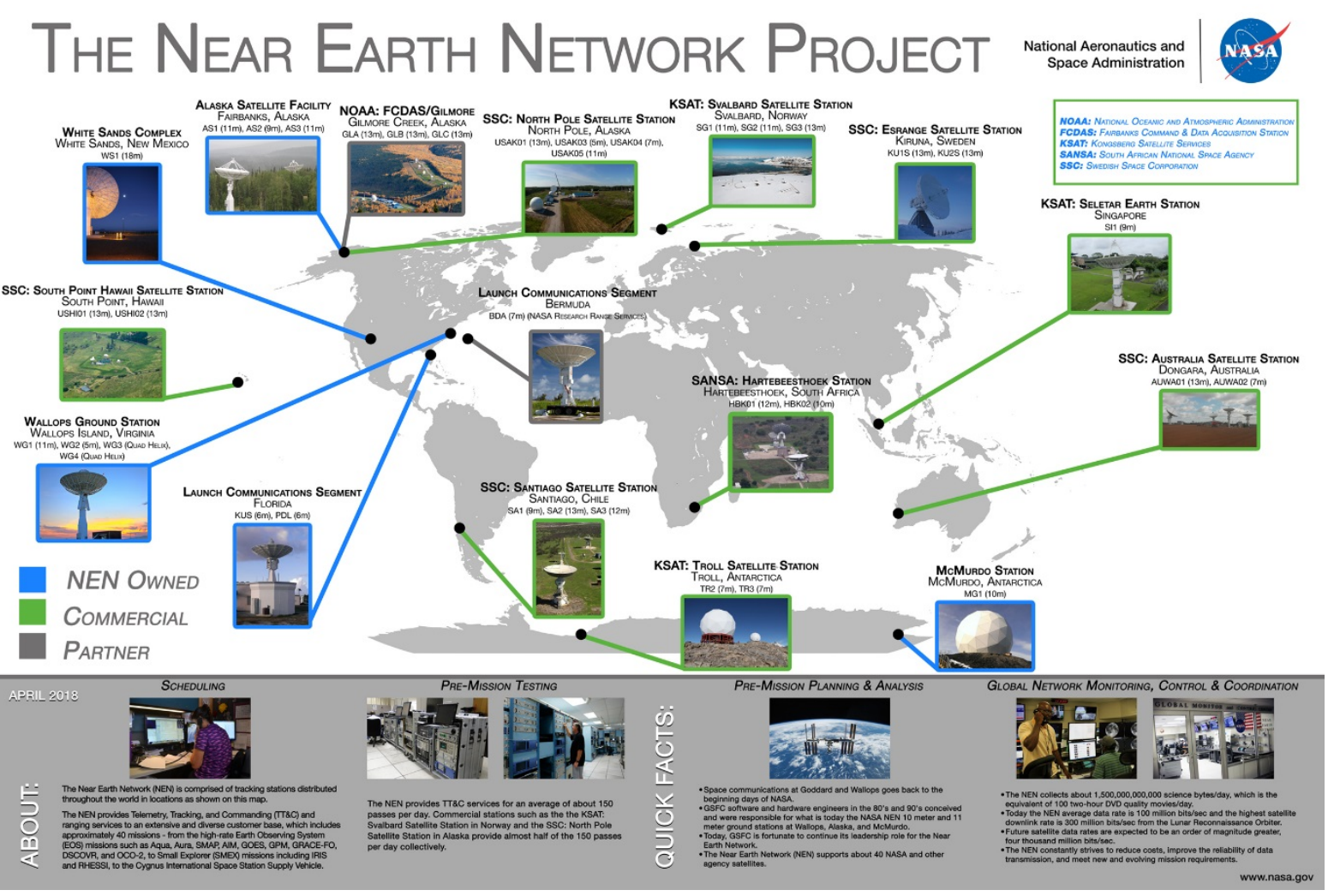

Fig. 2 The NEN Supports Orbits in the Near Earth Region from Earth to 2 Million Kilometers

In 2013 the NEN officially established the Launch Communications Stations Development Project to implement the new Launch Communications Segment (LCS) comprised to two permanent and identically configured ground stations: the new Kennedy Uplink Station (KUS) and a modernized tracking station at PDL. This paper will provide an overview of the LCS project, including the project history, architecture, and capabilities of each station. It will then present the three anticipated use cases of the LCS, which include:

1) Agile, tailored, robust launch, assent, and landing support

2) Orbital S-band communications services to near-Earth missions

3) Operational proving ground for Space Mobile Network concepts and technologies.

\section{Launch Communications Segment Overview}

\section{A. LCS History}

In September 2012, NASA KSC and the United States Air Force (USAF) Eastern Range released a joint Range Future State Definition (FSD) study that was developed by a team that included representation from NASA, Air Force Space Command (AFSPC), the Federal Aviation Administration (FAA), Space Florida, and supporting contractors. The report developed a vision for "[a]n East Coast launch support capability with NASA infrastructure and systems complementing the Air Force Eastern Range, using integrated, common processes to provide flexible, affordable, and responsive support to the multi-user community", including military, civil, and commercial users [3]. Two key stakeholders identified in the FSD study were the NASA Space Launch System (SLS) and the Orion Multi-Purpose Crew Vehicle (MPCV), the launch and crew vehicles that will support NASA's exploration vision. The FSD study issued several near- and long-term infrastructure modernization recommendations, two of which became driving operational capability requirements for NEN LCS.

In response to the FSD, the NEN began an initial period of pre-formulation concept development that led to a consensus regarding the basic ground architecture and concept of operations, as well as the driving technical, resource, and organizational requirements and constraints. The NEN project formally created the NASA LCS development project in 2013 to define, design, implement, and test modernized launch communications capabilities consistent with 
the FSD study vision and driven by the Exploration Mission-1 (EM-1) requirements, which encompass the SLS and Orion stakeholder mission requirements. As of early 2018, the LCS project has completed three of the four planned NASA systems engineering milestone reviews, with the fourth, the Operational Readiness Review (ORR), planned for late 2018, after which the two LCS stations will be operational.

\section{B. LCS Capabilities}

The LCS's two modern ground stations were designed to complement the U.S. Air Force's Eastern Range, enabling communications support to next-generation space missions and launch vehicles departing from, or returning to, the Florida spaceport. The LCS stations will provide the critical link between astronauts and mission controllers on crewed flights, and support launch vehicle telemetry and orbital tracking communications for robotic missions.

The NEN LCS is comprised of two permanent ground stations, KUS and PDL, which are each equipped with a single 6.1-meter antenna protected under a radome, as shown in Fig. 3. Both sites can provide a broad array of communications services. As part of a broader inter-agency collaborative agreement, the antenna systems were provided to NASA by the USAF for refurbishment and use at KUS and PDL, resulting in substantial LCS development cost savings. Originally the antenna systems were capable of S-band downlink and tracking only, but they have been modified by the project to support S-band uplink for command and voice data between mission controllers and the vehicle or crew.
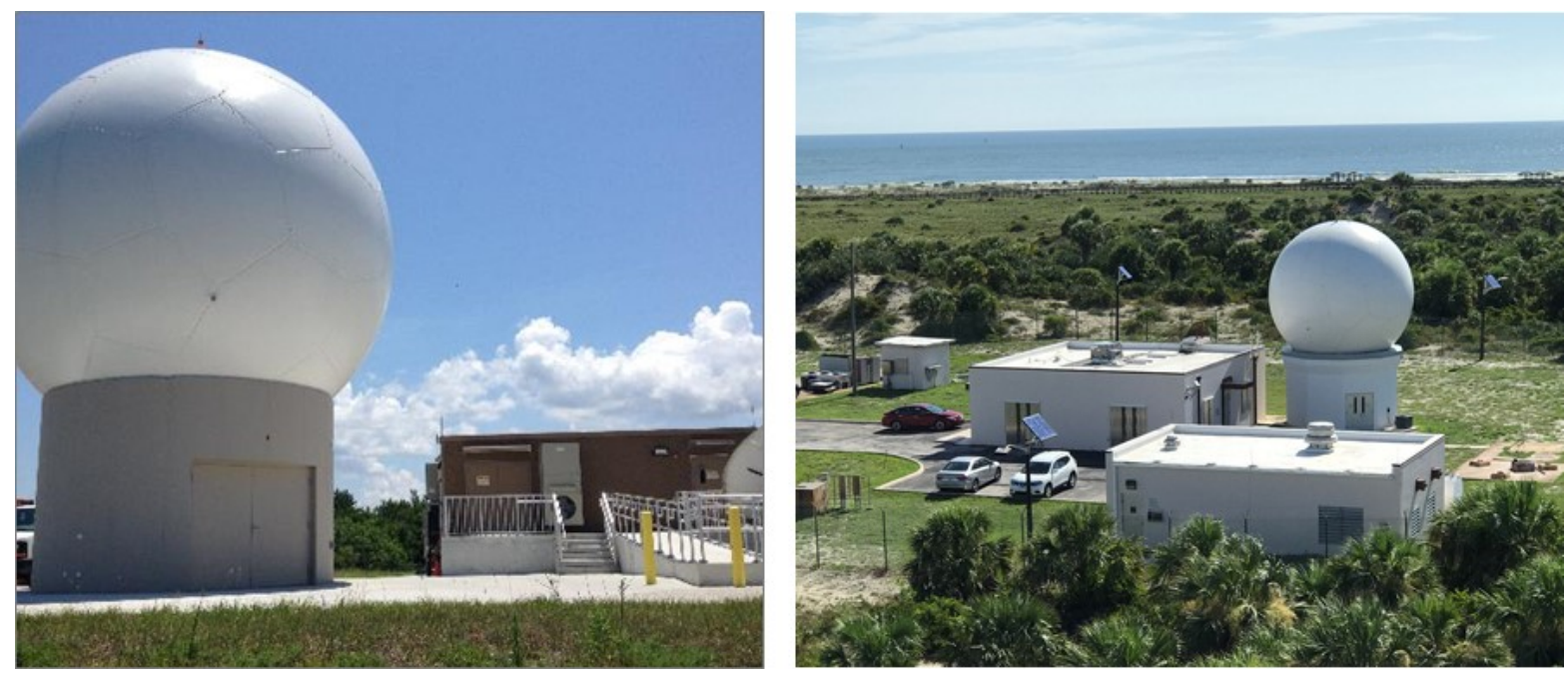

Fig. 3 (a) Kennedy Uplink Station (KUS) and (b) Ponce de Leon (PDL)

The ground stations feature all new state of the art signal processing and data handling components, including software defined radios compliant with Consultative Committee for Space Data Systems (CCSDS) data standards as well as Inter-Range Instrumentation Group (IRIG) 106 signals and data standards. The equipment provides the ability to support all the standard and advanced bandwidth efficient modulations and coding schemes currently used and recommended for future use by NASA missions, as well as many used by other civil, military, and commercial users.

KUS and PDL are equipped with an internal IP interface, through a fiber connection, with the Communications Distribution and Switching Center (CD\&SC) facility located at KSC that supports the sending and receipt of baseband data in IP formats. The NEN presence at the CD\&SC includes a Space Link Extension (SLE) Gateway that acts as the external interface to user mission ground systems. Real-time data and commands can be transferred using the CCSDS SLE format. Post-pass playback of mission data can be made available for post-support retrieval using the SLE offline mode.

Both stations are capable of being remotely monitored and controlled from the NEN's Global Monitor and Control Center (GMaCC) at the Wallops Flight Facility for routine events and pre-mission testing. Additionally, both KUS and PDL support standard service scheduling through the NEN Scheduling Office (NENSO). The Table 1 and Table 2 summarize the telemetry and command characteristics of KUS and PDL. 
Table 1 KUS and PDL S-Band Telemetry Characteristics

\begin{tabular}{|c|c|}
\hline Characteristic & Value \\
\hline Frequency & $2200-2395 \mathrm{MHz}$ \\
\hline $\mathrm{G} / \mathrm{T}$ & $\begin{array}{l}17.2 \mathrm{~dB} / \mathrm{K} \\
\text { (Assumes transmitter is up, clear sky conditions, and a } 5 \text { degree } \\
\text { elevation angle. Accounts for the following losses: radome loss, con- } \\
\text { scan/auto-track loss, tracking error loss for velocity }<=5 \text { deg/sec and } \\
\text { acceleration }<=5 \text { deg/sec }{ }^{2} \text {, and antenna to back end electronics loss) }\end{array}$ \\
\hline Polarization ${ }^{10}$ & $\begin{array}{l}\text { RHC or LHC } \\
\text { Diversity combination of RHCP/LHCP (pre/post detection) }\end{array}$ \\
\hline Telemetry Demodulation & $\begin{array}{l}\text { PM, FM, } \\
\text { PCM/PSK/PM, PCM/PM, PCM/FM, BPSK, QPSK, OQPSK } \\
\text { (SQPSK), SOQPSK, SQPN, AQPSK }\end{array}$ \\
\hline PM Modulation Index & 0 to 2.5 radians \\
\hline FM Deviation & $<=+/-500 \mathrm{kHz}$ \\
\hline Carrier Information Rate & $\begin{array}{l}100 \mathrm{bps} \text { to } 40 \mathrm{Mbps} \\
\text { *Maximum rate is modulation and coding dependent }\end{array}$ \\
\hline Carrier Line Coding & $\begin{array}{l}\text { NRZ: L,M,S } \\
\text { Bi } \Phi: \text { L, M, S } \\
\text { DBP: M, S } \\
\text { DM: M, S } \\
\text { R-NRZ } \\
\text { V35 }\end{array}$ \\
\hline Subcarrier Frequency & $\begin{array}{l}40 \mathrm{~Hz} \text { to } 128 \mathrm{kHz} \text { (Low Bandwidth Telemetry Mode) } \\
5 \mathrm{kHz} \text { to } 2 \mathrm{MHz} \text { (High Bandwidth Telemetry Mode) }\end{array}$ \\
\hline Subcarrier Information Rate & $\begin{array}{l}7 \text { bps to } 25 \text { kbps (Low Bandwidth Telemetry Mode) } \\
100 \text { bps to } 600 \text { kbps (High Bandwidth Telemetry Mode) } \\
\text { *Modulation and coding dependent }\end{array}$ \\
\hline Subcarrier Line Coding & $\begin{array}{l}\text { NRZ: L,M,S (Low or High Bandwidth Telemetry Mode) } \\
\text { Biథ: L, M, S (Low or High Bandwidth Telemetry Mode) } \\
\text { DBP: M, S (High Bandwidth Telemetry Mode) } \\
\text { DM: M, S (High Bandwidth Telemetry Mode) } \\
\text { R-NRZ (High Bandwidth Telemetry Mode) }\end{array}$ \\
\hline Decoding & $\begin{array}{l}\text { Viterbi } 1 / 2 \\
\text { Reed-Solomon }(255,223) \mathrm{i}=1 \text { to } 5 \\
\text { Concatenated (Reed-Solomon/Convolutional) } \\
\text { LDPC } 1 / 2 \\
\text { LDPC } 7 / 8,(8160,7136) \\
\text { Turbo Code } 1 \frac{12}{2}\end{array}$ \\
\hline Supported Data Formats & CCSDS and IRIG-106 \\
\hline
\end{tabular}

10 Post Detection Diversity Combining is available for the following modulations schemes: Video Mode (PM), PCM Mode (PCM/PM, PCM/BPSK, PCM/QPSK, or PCM/OQPSK). Pre Detection Diversity Combining is available for the following modulations schemes: Video Mode (PM, FM, Coherent/Non-Coherent FM), PCM Mode (PCM/PM, PCM/BPSK, PCM/QPSK, or PCM/OQPSK). 
Table 2 KUS and PDL S-Band Command Characteristics

\begin{tabular}{|c|c|}
\hline Characteristic & Value \\
\hline Frequency & $2025-2120 \mathrm{MHz}$ \\
\hline EIRP & $57 \mathrm{dBWi}$ (maximum) \\
\hline Polarization & RHC or LHC \\
\hline Command Modulation & $\begin{array}{l}\text { PM, FM, } \\
\text { PCM/PSK/PM, PCM/PM, PCM/FM, BPSK, QPSK, OQPSK } \\
\text { (SQPSK), } \\
\text { SS-UQPSK }\end{array}$ \\
\hline PM Modulation Index & $1-2.5$ radians \\
\hline FM Deviation & $<=+/-5 \mathrm{MHz}$ \\
\hline Carrier Data Rate & $\begin{array}{l}7 \text { bps to } 10 \mathrm{kbps} \text { (Low Bandwidth Command Mode) } \\
100 \text { bps to } 600 \mathrm{kbps} \text { (High Bandwidth Subcarrier Command Mode) } \\
100 \text { bps to } 1 \mathrm{Mbps} \text { (High Bandwidth PCM Command Mode) }\end{array}$ \\
\hline Carrier Data Format & $\begin{array}{l}\text { NRZ: L,M,S } \\
\text { Bi } \Phi: \text { L, M, S } \\
\text { R-NRZ }\end{array}$ \\
\hline Subcarrier Frequency & $\begin{array}{l}40 \mathrm{~Hz} \text { to } 100 \mathrm{kHz} \text { (Low Bandwidth Command Mode) } \\
5 \mathrm{kHz} \text { to } 2 \mathrm{MHz} \text { (High Bandwidth Subcarrier Command Mode) }\end{array}$ \\
\hline Subcarrier Data Rate & $\begin{array}{l}7 \text { bps to } 10 \mathrm{kbps} \text { (Low Rate Command Mode) } \\
100 \text { bps to } 600 \mathrm{kbps} \text { (High Rate Command Mode) }\end{array}$ \\
\hline Subcarrier Data Format & $\begin{array}{l}\text { NRZ: L,M,S } \\
\text { Bi } \Phi: \text { L, M, S } \\
\text { R-NRZ }\end{array}$ \\
\hline Coding & Convolutional $1 / 2$, LDPC $1 / 2$ \\
\hline
\end{tabular}

\section{Use Case \#1: Launch, Ascent, and Landing Support}

The first LCS use case is to provide agile, tailored, and robust launch, ascent, and landing communications solutions to the Florida spaceport. This includes support to EM-1 (SLS and Orion), the driving LCS mission, as well as legacy and expected future civil, commercial, and military launch communications users. The support solutions can be based solely on the two permanent LCS stations or could be extensible through host-tenant arrangements with the USAF and deployable assets in partnership with the NASA Wallops Research Range to meet mission specific requirements.

\section{A. Modernization Activity and Driving LCS Support Requirements}

Following the end of Shuttle Program and the decommissioning of MILA and PDL there were multiple efforts conducted to define and develop NASA's next generation launch communications services out of Florida. Several market analyses and feasibility studies investigating concepts were performed during the Constellation Program prior to its cancellation in 2009, and as part of the KSC's follow-on efforts to transform itself into a 21st century multi-user spaceport. The 2012 FSD study to analyze the market needs of current and future launch systems and to assess the operational deficiencies of the Eastern Range infrastructure eventually led to development of the LCS driving requirements, shaping the early stages of the LCS project. The FSD study team issued multiple near-term and longterm recommendations. Two near-term recommendations drove operational capability requirements for the NEN LCS: (1) Support telemetry link data rates of at least $20 \mathrm{Mbps}$, and (2) S-band uplink capability to vehicle and/or crew. Both recommendations and the associated requirements were driven by EM-1. A NASA-USAF Memo of Understanding (MOU) was signed in late 2013 authorizing cooperation for the NEN LCS project [4]. The MOU officially levied additional requirements on LCS to support Eastern Range launch vehicle requirements (i.e. IRIG-106 data formats, as well as modulation and coding schemes for all Eastern Range supported launch vehicles). The MOU also captured the USAF agreement to transfer USAF equipment to NASA, including two TAA-20 (6.1-meter) telemetry antenna systems now installed at KUS and PDL. 


\section{B. LCS Site Placement for Launch/Landing Support}

The locations of the two permanent LCS ground stations, KUS and PDL, were strategically determined. Two sites are required to ensure continuous communications coverage during launch and ascent. Existing Shuttle-era infrastructure and land use agreements, as well as operationally validated site performance, made PDL a cost effective and low risk site location choice. As a completely new site, criteria for KUS site selection included line-of-sight coverage to the active KSC and Cape Canaveral Air Force Station (CCAFS) launch pads, which would be used for EM-1 as well as other military, civil, and commercial launch vehicles, in addition to having a view of the Shuttle Landing Facility (SLF). Located at the former site of the KSC Environmental Health Facility (EHF), KUS was constructed approximately $5 \mathrm{~km}$ south of the Vehicle Assembly Building (VAB). This location provided optimal lineof-sight coverage to Launch Complex 39B (to be used for SLS), Launch Complex 39A and Space Launch Complex 40 (used for SpaceX), Space Launch Complex 41 (used for United Launch Alliance Atlas V), Space Launch Complex 46 (used for Athena) as well as other active launch pads, as shown in Fig. 4. KUS line-of-sight coverage to the launch pads is protected by the KSC Master Plan to ensure KUS will be able to support the growing and evolving needs of Florida spaceport users.

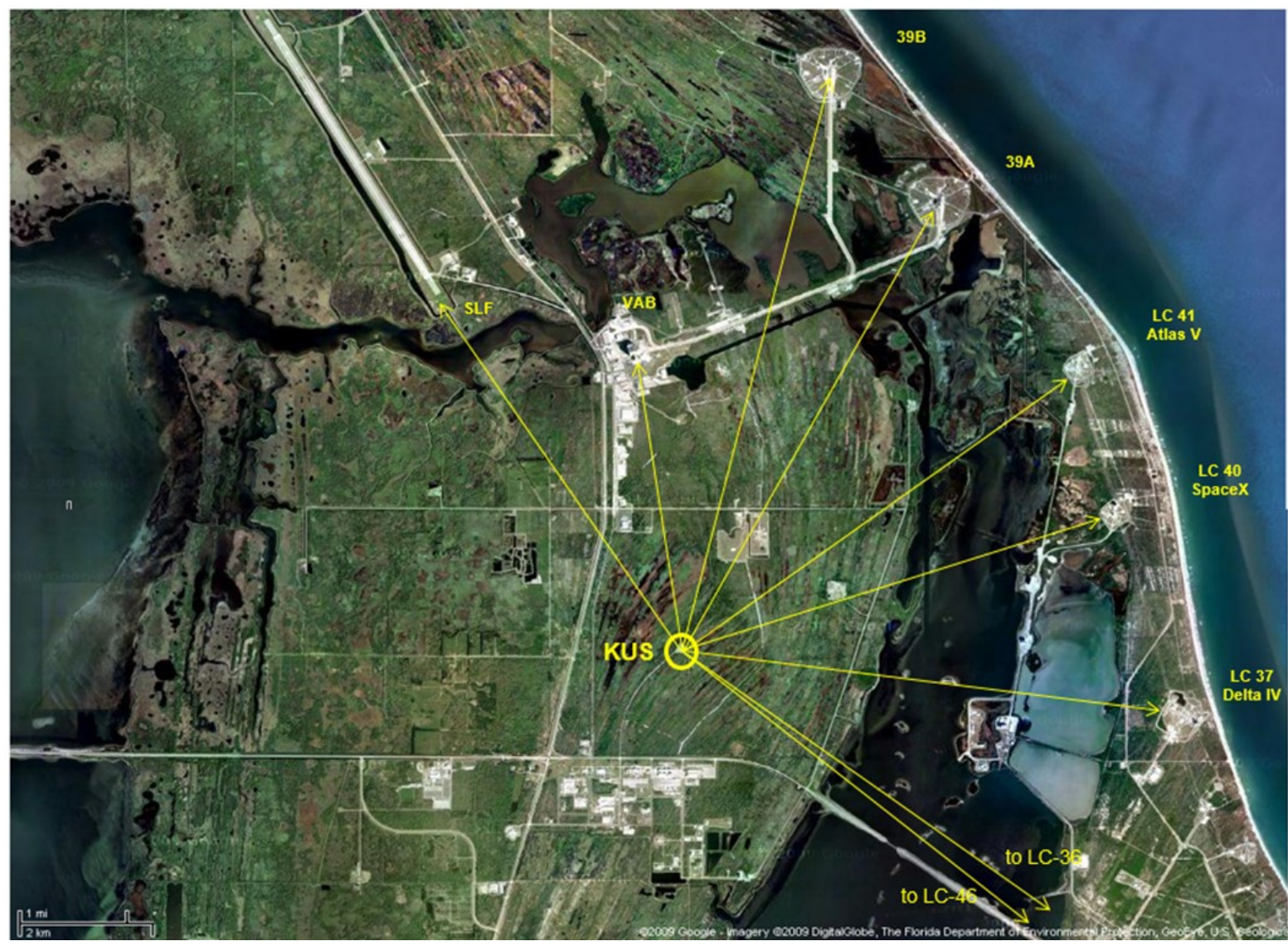

Fig. 4 KUS Line of Site to VAB and Launch Pads

\section{EM-1 Overview and Link Allocations}

EM-1 will be the first integrated flight of NASA's flagship human exploration systems. It will consist of the SLS core stage, Orion MPCV, and a second stage rocket known as the Interim Cryogenic Propulsion Stage (ICPS). SLS is NASA's flagship heavy-lift launch vehicle. It is anticipated to carry humans to deep space, the Moon and Mars, and to increase accessibility to the outer solar system for robotic missions. SLS is designed to advance through different block configuration, with each incremental block configuration increasing its power and lift capability. The Orion MPCV is the exploration vehicle that will carry the crew to space, provide emergency abort capability, sustain astronauts during phases of their missions, and provide safe re-entry from deep space. For the EM-1 test flight, astronauts will not be on-board. ICPS will provide the thrust needed to send the unoccupied Orion capsule into lunar orbit on EM-1. However, for EM-2 and beyond ICPS will be replaced with a new more powerful Exploration Upper 
Stage (EUS). The LCS project anticipates supporting EUS telemetry during launch and ascent, as well as during a checkout phase prior to Orion/EUS departure on a Lunar transfer orbit from as many as seven NEN, partner, and deployable ground stations in addition to KUS and PDL.

The SLS core stage has two $20 \mathrm{Mbps}$ (encoded) telemetry downlinks. One of these links must be processed and distributed to end users with very low latency. The other link contains data that supports the flight test and engineering objectives associated with characterizing and understanding SLS design performance. These data are used for postflight analyses and may be delivered to the mission users up to 72 hours following the launch. The Orion MPCV Sband uplink includes voice data from mission control. Orion also has a downlink that includes crew voice as well as crew systems and vehicle telemetry. The ground stations will have low latency terrestrial network connectivity to mission control to support the Orion MPCV uplink and downlink.

\section{LCS Tailored Ground Architecture for EM-1}

For complete and comprehensive support of the EM-1 requirements, the two LCS station architecture of KUS and PDL will be augmented and tailored through agreements and partnerships with both the USAF and the NASA Wallops Range. A five-site ground architecture is necessary to support EM-1 communications requirements, and consists of: KUS and PDL, USAF Tel-IV and Jonathan Dickinson Missile Test Annex (JDMTA) stations and the NASA Wallops Range's downrange Bermuda (BDA) station. Fig. 5 shows the EM-1 support architecture.

The Orion MPCV S-band uplink and downlinks will be supported at the NASA KUS and PDL stations. This allocation is consistent with the historical arrangement for human space flight launch communications services and avoids costly retrofitting of the existing antenna subsystems at Tel-IV and JDMTA, which do not presently support uplink services. The two-high data rate SLS core stage telemetry links will also both be supported by the KUS and PDL stations. This allocation leverages the low latency terrestrial network connectivity required to support the Orion MPCV links, and minimizes the scope and complexity of the required terrestrial network infrastructure. The two USAF stations, Tel-IV and JDMTA, will provide S-band downlink support for the ICPS link and will support GPS metric tracking and range safety functions. As the vehicles move downrange, Orion and ICPS will transition from ground support to NASA's Space Network. However, the two SLS core stage telemetry links must be supported from the BDA downrange ground station due to their high data rates. During the LCS development phase, it was discovered that the existing Wallops Range signal processing equipment was unable to support the advanced signal characteristics and data rates of the SLS core stage telemetry links. The project was able to quickly adapt by replicating the signal processing and data dissemination designs from KUS and PDL. As a result, the BDA station uses a Wallops Range owned and operated transportable antenna feeding signals to NEN developed and operated backend electronics remotely monitored and controlled from the NEN GMaCC. The EM-1 ground support architecture illustrates how LCS has formed cross-Agency engineering and operational partnerships, resulting in a cost-effective, agile, robust, and tailored communications solution, exemplifying the FSD vision and serving as a model for future Florida spaceport users. 


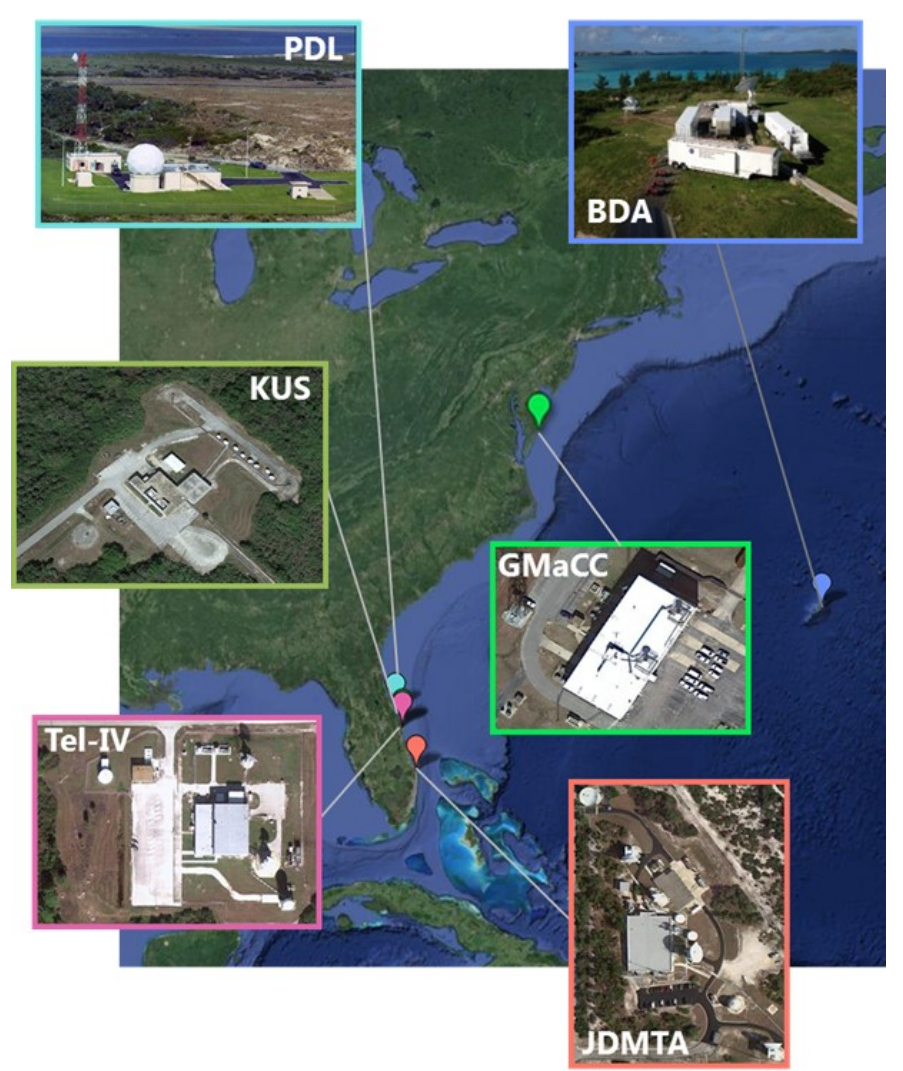

Fig. 5 LCS Tailored (Augmented) Ground Architecture for EM-1

\section{E. Additional LCS Capabilities}

As a result of the LCS project, many new capabilities have been incorporated into the NEN standard design components. These include spread spectrum modulation support, Low Density Parity Check (LDPC) error correction codes, support for IRIG-106 data formats, standardized Space Link Extension user interfaces, and a more robust implementation of the remote monitor and control software.

Another new capability, which has its roots in Shuttle support, is the inaccurately named "Best Frame Selector" (BFS). During the Shuttle era, a Best Source Select (BSS) system was used to ensure the ground station receiving the strongest signal was used for operational control and monitoring. For Shuttle launches the signal levels from MILA, PDL, Wallops, and other stations were evaluated during flight. As the vehicle traversed the coverage areas of various stations, a manual handover was performed. With LCS, all telemetry streams are sent to a centralized network location and algorithmically merged to form a single data stream. Effectively, it makes multiple telemetry stations appear as a single source to the end user, greatly reducing operational complexity and more efficiently using the terrestrial network bandwidth. The BFS algorithm simplifies operations by not disseminating data frames that have uncorrectable errors induced by plume effects (e.g., attenuation and diffraction model fades and scintillation), multipath, antenna pointing errors, or Radio Frequency Interference. However, the best frame select algorithm does not enhance the error correction code performance. Rather, the BFS provides the user with a non-repeating complete set of all successfully decoded data received by the connected ground stations (i.e., KUS, PDL, BDA, or other mission-specific stations). A notional timeline of the EM-1 Best Frame Select concept is shown in Fig. 6. 


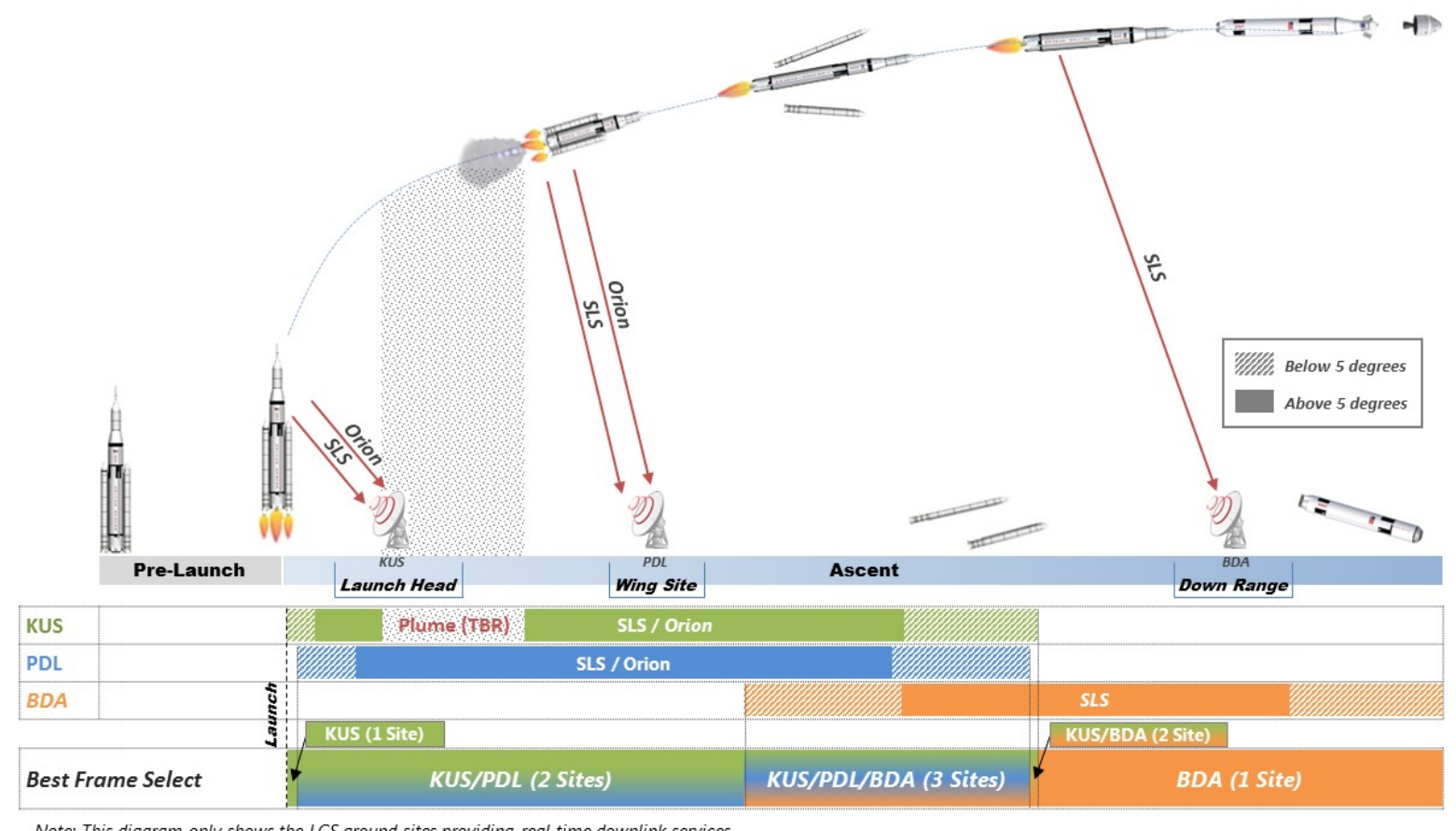

Fig. 6 NEN LCS EM-1 Real Time Telemetry "Best Frame Select" CONOPS

\section{F. Use Case 1 Summary}

KUS and PDL were designed to exemplify the FSD vision of NASA systems complimenting the Eastern Range and providing flexible, affordable and responsive services to the multi-user community. LCS provides unique S-band uplink capabilities for command or voice data and delivers a modernized communications solution for launch vehicles departing or returning to the Florida spaceport through the architecture and capabilities of KUS and PDL, or a tailored architecture augmented through host-tenant arrangements, partnerships and standard design components.

\section{Use Case \#2: Orbital S-band Communications Services to near-Earth Missions}

The second LCS use case is to provide orbital S-band communications services to near-earth users. The NEN traces its roots back to NASA's early communication networks, the STADAN and MSFN, which consisted of worldwide ground stations capable of providing communications services to robotic and human spaceflight missions. Around the same time, NASA also operated the DSN, based out of the Jet Propulsion Laboratory (JPL), which primarily focused on providing tracking, telemetry, and command to unmanned lunar and planetary spacecraft. In the 1980's, NASA introduced a new network, consisting of a fleet of communications satellites in GEO orbit, designated Tracking and Data Relay Satellite System (TDRSS). The TDRSS together with ground terminals located in White Sands, New Mexico, and Guam as of 1990's, made up the NASA SN. The SN can provide continuous global coverage to user missions from launch to LEO orbit.

The Space Communications and Navigation Program oversees NASA's three space communications projects: the NEN, DSN, and SN. The SCaN network is comprised of a set of Earth ground stations and space relays depicted in Fig. 7. Each network satisfies particular user needs in a complimentary fashion. In addition to its historical role in support of the Human Space Flight Program, the NEN provides direct-to-earth communications services primarily to satellites in Earth orbit, Lunar orbit, and within a service volume spanning out to 2 million kilometers. 


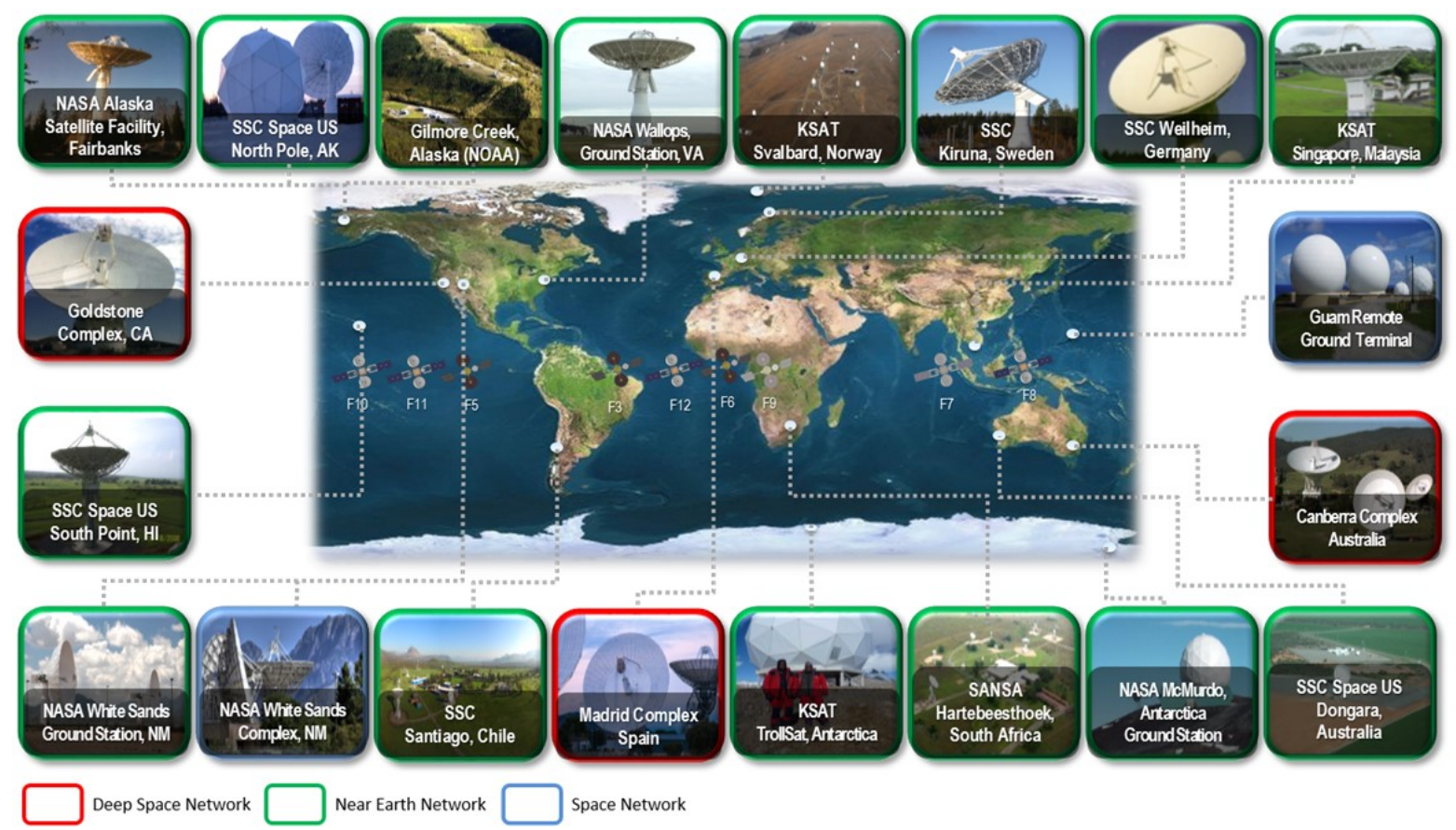

Fig. 7 SCaN Network

\section{A. LCS Role in the Broader Near Earth Network}

The launch communications use case was the principal driver in LCS performance requirements. However, KUS and PDL were designed to be compatible with the larger SCaN Program and NEN Project capability, operations, sustainment, security and programmatic architecture as well. SCaN maintains a Program (Level 2) Systems Requirement Document (SRD) that captures functional and performance requirements and flows them down to each of the three projects. The NEN maintains a Level 3 SRD, that traces to the SCaN SRD and elaborates and expands on the NEN requirements. The LCS development project derived Level 4 and below requirements, ensuring compliance with the broader project and program architecture. The LCS stations utilize the designs, components and remote operations concepts proven in other NEN orbital satellite tracking stations. The use of common commercial technologies and adherence to international data standards and protocols ensures that KUS and PDL can support the common modulations, coding, and other parameters necessary to service NEN S-band users. KUS and PDL also interface to the common NEN scheduling, logistics, safety, security, and other systems.

\section{B. LCS Orbital Tracking Readiness and Availability}

EM-1 is expected to launch in December 2019. This will be followed by EM-2 in late 2022, after which each subsequent EM launch will occur 1 year later (e.g., 2023, 2024, etc.). It is anticipated that LCS will support all crewed Orion missions, and all human and robotic SLS missions. Validation testing and shadow operations for nearly all Eastern Range launches began in 2017 at KUS and in 2018 at PDL. KUS and PDL are expected to provide backup support to the Orion Ascent Abort-2 (AA-2) in April 2019. Launch communications support to Florida spaceport users is the primary use case. However, due to the relatively low frequency of launch events as compared to orbital tracking opportunities, and the automated station re-configuration capabilities, it is anticipated that preliminary orbital tracking support may be available prior to EM-1, with routine orbital tracking operations expected to commence following EM-1 even in a scenario of frequent launch events. KUS and PDL are being included in the NEN mission planning and integration processes to provide routine S-band communications support to NEN orbital users.

\section{Use Case \#3: Operational Proving Ground for Space Mobile Network Concepts and Technologies}

The third LCS use case is to serve as an operational proving ground for future space communications operations concepts and technologies. KUS and PDL have radically reduced operations and sustainment costs as compared to the Shuttle-era ground stations. This is a result of many factors, including the miniaturization of electronic components, software-defined functional consolidation enabled by Field Programmable Gate Array (FPGA) and commodity processing resources, infusion of software-enabled automation technologies, widespread adoption of international 
signal and data standards, and the emergence of a competitive market for commercial space communications components. In the never-ending quest to achieve more with less, LCS will play a crucial role in NASA's space communications innovation strategy.

\section{A. NASA's Space Mobile Network Architectural Framework}

Inspired by the concepts and technologies driving innovation in terrestrial wireless mobile networks, NASA has introduced the Space Mobile Network (SMN) architectural framework [5]. The SMN framework broadens the scope of space communications innovation from a traditional focus on predictable point-to-point physical and link layer functions to include networking and application layer innovations to address dynamic and responsive service acquisition and execution scenarios, reduce user burden, and increase efficiency and effectiveness [6]. An overview of the SMN architecture framework is provided below in Fig. 8.

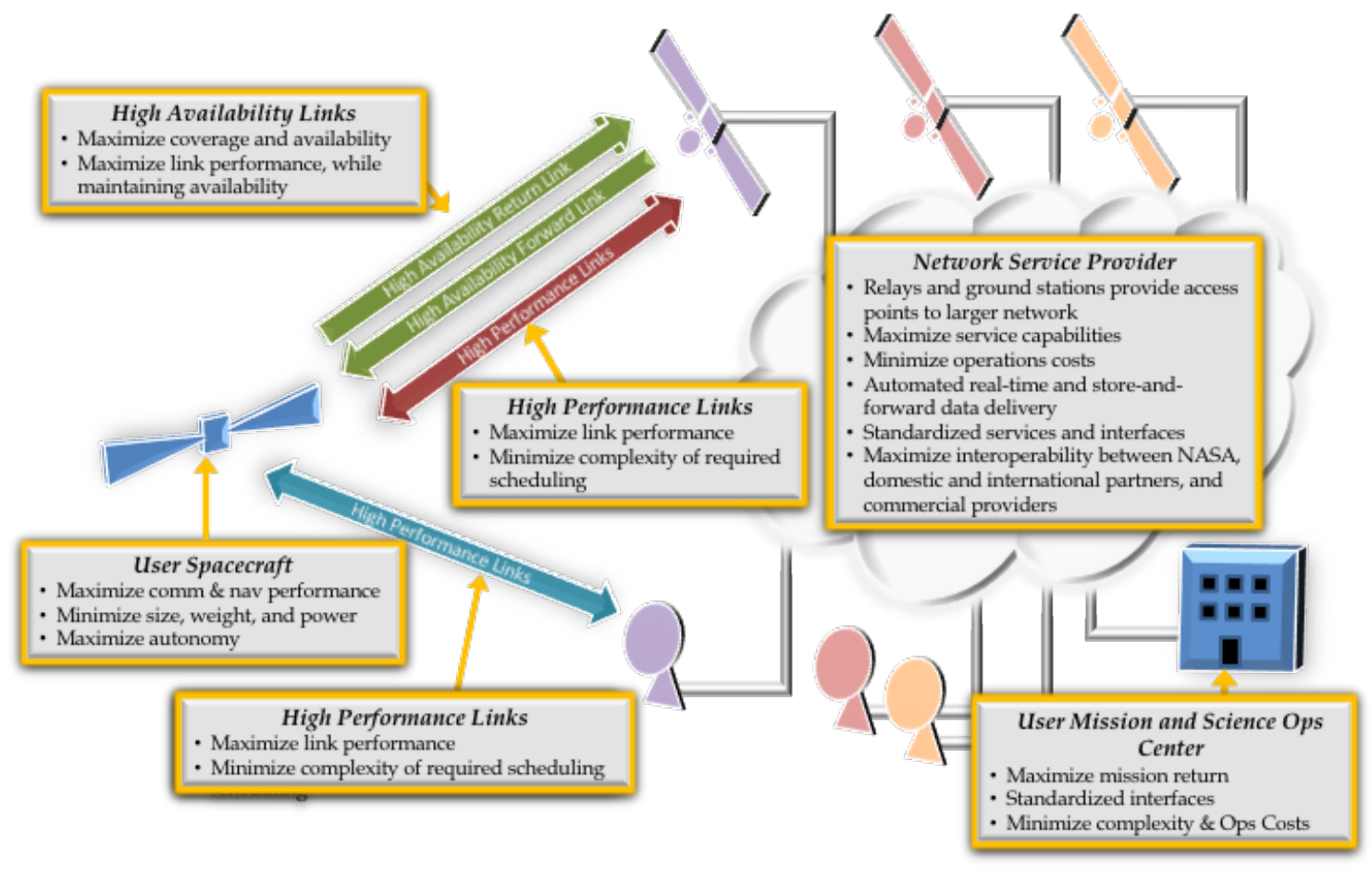

Fig. 8 Space Mobile Network Architectural Framework

\section{B. NEN LCS Space Mobile Network Innovations}

The Near Earth Network is unique among NASA space communications projects for its seamless integration of NASA owned and operated ground stations with university, international partner, and commercial stations. The NEN's ability to routinely and rapidly form partnerships in response to mission needs for greater performance, resiliency ${ }_{2}$ or efficiency is demonstrated by the augmented LCS ground architecture for EM-1. The NEN played an important role in fostering the emergence of a commercial market for space communications operational services and has been an early adopter of commercially sourced software-defined space communications technologies for its NASA owned stations. For these reasons, the NEN, and LCS in particular, are poised to lead many Space Mobile Network innovations. Key areas of emphasis for LCS experimentation and operational validation include User Initiated Services, Delay Tolerant Networking, ground-based phased array concepts for increased performance (multi-aperture) or availability (multi-element), adaptive and autonomous link configurations, processing resource virtualization, software defined networking, and emerging cybersecurity and distributed resource allocation applications for blockchain technologies. 


\section{Conclusion}

As described in this paper, the NEN LCS driving mission is to provide unique and complimentary launch communications services to the Florida spaceport. This includes support for the bi-directional S-band link with the Orion crew capsule and two high rate S-band telemetry links from the SLS core stage for EM-1. The advanced EM-1 mission requirements envelope legacy and expected future civil, commercial, and military launch communications users. The KUS site selection also considered potential future support for vehicles returning to the launch area or the Shuttle Landing Facility runway. When not providing launch communications testing or operational support, the NEN LCS will provide S-band tracking, telemetry, and command services to NEN orbital satellite mission users. Finally, the NEN LCS will provide a proving ground for demonstrating advanced Space Mobile Network communications concepts and technologies.

The collaborative and multi-faceted aspects of LCS are represented in its mission patch, illustrated in Fig. 9 below. The primary organizations responsible for the development and support of LCS are listed along the outer ring. In clockwise order, the Goddard Space Flight Center (GSFC) is responsible for oversight and management of LCS development and operations. Kennedy Space Center provided the development funds for the LCS project as part of its $21^{\text {st }}$ century spaceport transformation initiative and serves as the host site for Kennedy Uplink Station. The Ponce de Leon (PDL) wing site location at New Smyrna Beach incorporates the anchor from the town's community seal. The anchor symbolizes costal community resiliency and is fitting to PDL's role ensuring continuous communications coverage in the LCS architecture [7]. LCS depends on collaborative relationships, complimentary operations and support provided by several U. S. Air Force (USAF) organizations, including the $45^{\text {th }}$ Space Wing and the Space and Missile Systems Center. Finally, the NEN engineering and operations teams are based at Wallops Flight Facility (WFF), as is the Wallops Range organization, our partner for the augmented Bermuda downrange station in the EM1 ground architecture. Yellow stars along the stylized east cost of the United States represent the permanent LCS operational sites: KUS, PDL and the Wallops Global Monitor and Control Center. The red, orange and yellow vector encircled by an ellipse and topped with a jagged star represent the launch and orbital flight regimes supported by LCS. This element draws inspiration from the astronaut insignia and the Commercial Crew Program logo, recognizing LCS's role enabling crew voice communications with ground controllers. The stylized white ground antenna in the center of the patch is incorporated from the NEN project logo. The overlaid and rotated letter C is in alignment with the ground antenna to suggest they tracking the star. The launch plume vector and star exceed the circular boarder of the patch to symbolize continuity with the past standard of excellence set by MILA during the Apollo and Space Shuttle Programs, and LCS's contribution to the continued exploration and development of space.

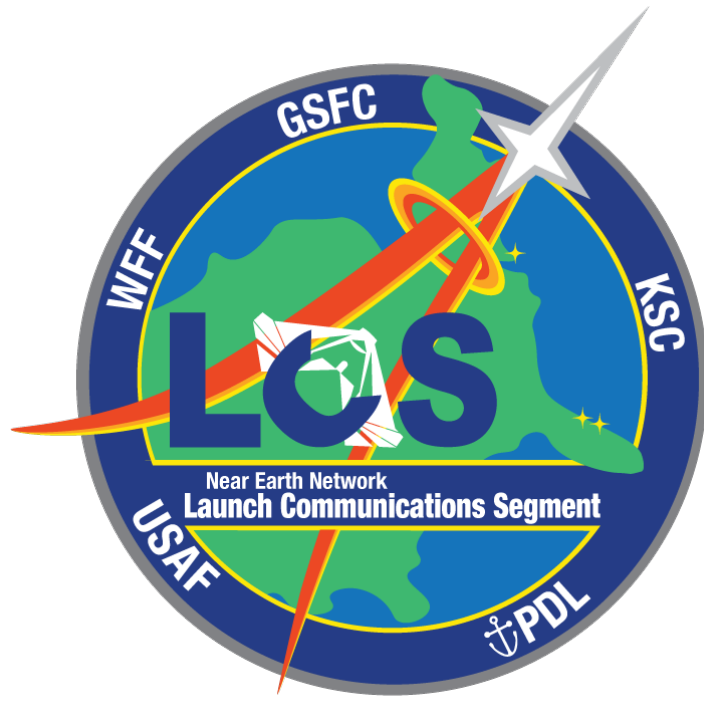

Fig. 9 The Collaborative and Multi-Faceted Aspects of LCS are Represented in its Mission Patch 


\section{Acknowledgments}

C.J. Roberts would like to express gratitude for the tremendous tenacity, competence, and dedication of the LCS development team, whose tireless efforts drove continuous progress despite change, uncertainty and adversity. On behalf of the LCS development team, C.J. Roberts would also like to thank the Near Earth Network Project, the Space Communications and Navigation Program, the Kennedy Space Center Ground Systems Development and Operations Program, the U.S. Air Force, the Cross-Program Integrated Communications, Network and Tracking Integrated Product Team and the Human Space Flight Network Support Group for resources and support enabling our contribution to the continued exploration and development of space. D. McCormick and C. Roberts would like to thank Daniel Baird for his artistic skill in developing the LCS mission patch.

\section{References}

[1] Roberts, C., Carter, D., Hudiburg, J., Tye, R., Celeste, P., and Peskett, P., "Evolving the NASA Near Earth Network for the Next Generation of Human Space Flight", presented at the SpaceOps 2014 Conference, Pasadena, California, 2014.

[2] Tsiao, S., Read You Loud and Clear!: The Story of NASA's Spaceflight Tracking and Data Network, National Aeronautics and Space Administration History Division, SP-2007-4233 Washington, DC, 2008.

[3] Ground Systems Development and Operations Program, "Range Future State Definition Study, Final Report” NASA Kennedy Space Center, FSD-008, 16 Sep. 2012.

[4] Whelan, M., and Gerstenmaier, W., "Memorandum of Understanding between the Air Force Space Command (AFSPC) and the National Aeronautics and Space Administration (NASA), Human Exploration and Operations Mission Directorate (HEOMD) on Telemetry System Upgrades at TEL-4 (TAA-20) and Jonathan Dickinson Missile Tracking Annex (JDMTA) on the USAF Eastern Range (ER)," Nov. 2013.

[5] Israel, D., Heckler, G., and Menrad, R., "Space Mobile Network: A Near Earth Communications and Navigation Architecture", presented at the IEEE Aerospace Conference, Big Sky, MT, United States, 2016.

[6] Israel, D., Roberts, C., Morgenstern, R., Gao, J., and Tai W., "Space Mobile Network Concepts for Missions Beyond Low Earth Orbit", presented at the AIAA 15th International Conference on Space Operations, Marseille, France, 2018.

[7] In Official City Website of New Smyrna Beach Florida, from https://www.cityofnsb.com 